\title{
IPv6 Performance Analysis Based on Protocol and Tunnel Transition
}

\author{
A. Rajkumar ${ }^{1}$, G. Kannan ${ }^{2}$ \\ ${ }^{1}$ M.E. Communication Systems, Department of Information and Communication Engineering \\ Anna University, P.R Engineering College, Thanjavur, 613403 \\ ${ }^{2}$ Professor, Department of Electronics and Communication Engineering, Anna University, \\ P.R Engineering College, Vallam, Thanjavur, 613403
}

\begin{abstract}
Based on the migration mechanism from IPv4 to IPv6, the performance of three kinds of mechanism options, double-stack protocol, ISATAP tunneling and 6 to 4 tunneling technique are analyzed and tested. The result shows there are some performance advantages on double stack protocol mechanism IPv6 network than IPv4, IPv6 ISATAP tunnel and IPv6 6to4 tunnel network.
\end{abstract}

Keywords: double-stack protocol, tunnel technique, IPv6, throughput, round-trip delay

\section{Introduction}

Due to IPv4 network suffering more and more problems, especially the lack of address space as well as the network Security flaws, the next generation IPv6 network research is caught to be focused [1]. The IPv6 has solved IP address crisis, which expands IP addresses from 32-bit to 128-bit. There is limitation of compatibility between IPv6 and IPv4, therefore, transition mechanism from IPv4 to IPv6 is studied widely, mainly focuses on dual stack mechanism and the tunnel mechanism two options. This paper describes the principle of transitional mechanism, makes a comparison and analysis by testing the performance of IPv6 based on commonly used transition mechanism [2][3].

\section{Transition Mechanism}

\subsection{Dual-Stack Protocol}

In dual-stack, all hosts/routers maintain both protocol IPv4 and IPv6 stacks. Dual stack hosts/routers are able to communicate with not only IPv6 system, but also IPv4 system. The dual stack hosts use IPv6 address while communicating with IPv6 hosts, and use the IPv4 address while communicating with IPv4 hosts. Applications choose between using IPv6 or IPv4 with the application selecting the correct address based on the type of IP traffic and particular requirements of the communication.

\subsection{Tunnel Mechanism}

Another transition to IPv6 is using tunnel technique. The element of this approach is to encapsulate IPv6 datagram into IPv4 by dual-stack protocol routers while IPv6 datagram entering IPv4 network, and to make the IPv6 packet become part of IPv4 packet. Then IPv6 packets start transition within IPv4 tunnel network. At the point of the IPv4 datagram leaves the tunnel of IPv4 network, the dualstack routers will forward data, the original IPv6 packet, to the IPv6 protocol stack. The essence of tunnel technique is IPv6 packets will be encapsulated in IPv4 packets, using the existing communication problems between. IPv4, IPv4 transmission path as IPv6 data link layer, can be regarded as a point to point virtual link.

\subsubsection{6to4 Tunnel Mechanism}

The core idea of 6to 4 tunnel mechanism is that site address prefix contains IPv4 tunnel port address, a mapping created between IPv4 address of site border routers and IPv6 address of intra-site hosts, and directly uses IPv4 address of site border router as part of IPv6 address prefix of intra-site hosts.

\subsubsection{ISATAP tunneling Mechanism}

Intra-site Automatic Tunnel Addressing Protocol (ISATAP) is designed for the intra-site scope. With ISATAP, the intrasite IPv4 network is viewed as a link layer for IPv6 and other nodes in the intra-site network are viewed as potential IPv6 hosts/routers. An ISATAP address is formed with its own interface identifier. After that, the ISATAP hosts can connect each other via the IPv6-in-IPv4 tunnel with ISATAP address.

\section{Performance Test Design}

In this paper, the throughput and round-trip delay are tested by comparing IPv6 to IPv4 network performance as well as IPV6 network performances under those three mechanisms above. Based on test results, IPV6 network performance advantage is proved.

\subsection{Testing structure}

In Figure 1, PC1 and PC2 are tested transmitter and receiver running with Windows operation system. Due to limited resource, routers are simulated with high-performance PC. Choosing active testing method, that is, sent specific strength data packets from PC1 to PC2 through the network to test [8]. 


\section{International Journal of Science and Research (IJSR) \\ ISSN (Online): 2319-7064}

Index Copernicus Value (2013): 6.14 | Impact Factor (2015): 6.391

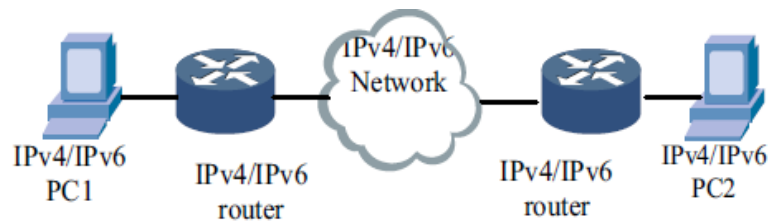

(a) Dual stack protocol

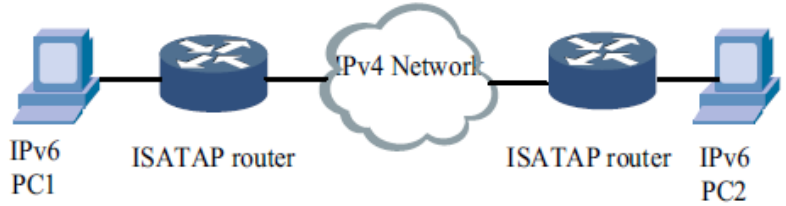

(b) ISATAP tunnel mechanism

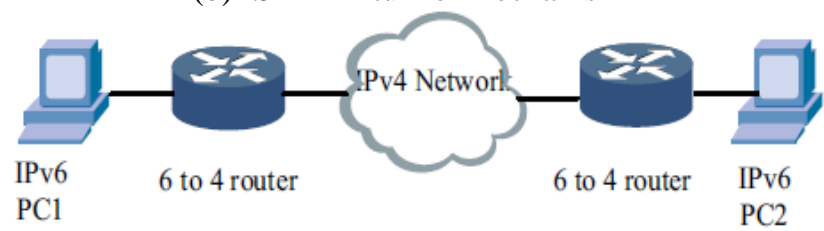

(c) 6to4 tunnel mechanism

Figure 1: Testing Structure

\section{Test Analysis}

\subsection{Throughput Test}

Throughput is defined as the amount of packet data that is transmitted over the entire path per time unit. The throughput is calculated from the formula $\mathrm{T}=\mathrm{P} / \mathrm{L}$ where $\mathrm{T}$ represents the throughput, $\mathrm{P}$ represents the transferred data size, and L represents the time cost in transfer. TCP protocol is used in the throughput test, test data packet's payload size from 64 bytes to 1408 bytes, and each group's result takes the average value of 10 times test. Figure 2 shows comparison result of IPv6 and IPv4 network throughput under the dual-stack protocol. Figure 3 shows dual stack IPv6 network throughput comparison between ISATAP tunnel mechanism and 6 to 4 tunnel mechanism.

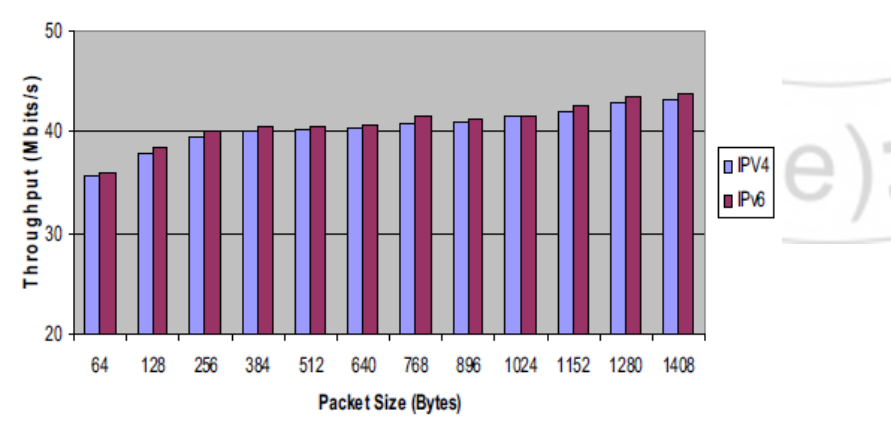

Figure 2: IPv6 and IPv4 network throughput comparison

In Figure 2, in dual-stack mechanism, TCP/IPv6 throughput increases accompanying with the packet payload size increasing, and shows larger volume than TCP/IPv4 throughput under the same condition. Figure 3 is also based on the same test condition, throughput of 6to4 and ISATAP tunnel mechanism look similar. But throughput of dualstack mechanism is more than the other two.

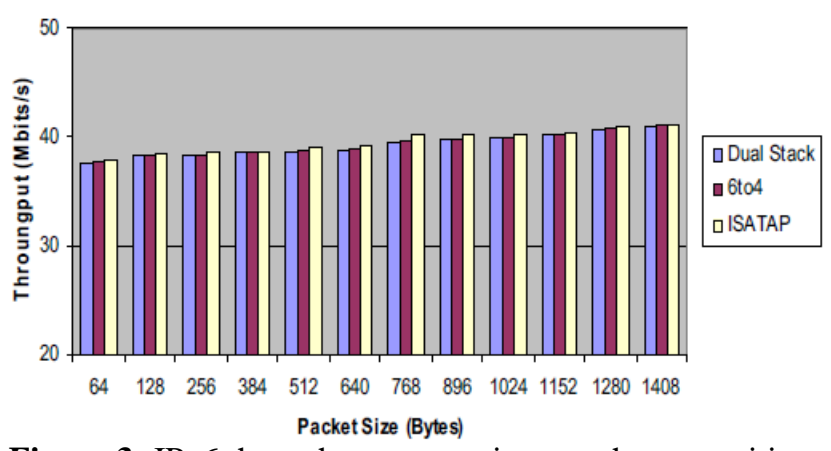

Figure 3: IPv6 throughput comparison on three transition mechanisms

\subsection{Round-trip delay test}

IPv6 and IPv4 network round-trip delay test uses ICMPv6 and ICMP protocol respectively, test data packet size is 1024 bytes and each group test results obtain from the average of 100 tests. Figure 4 shows 50 groups result of IPv6/IPv4 network for dual stack mechanism. Figure 5 is for IPv6 network round-trip delay comparison of dual-stack mechanism, ISATAP and 6to4 tunnel mechanism.

\section{RTT Compare}

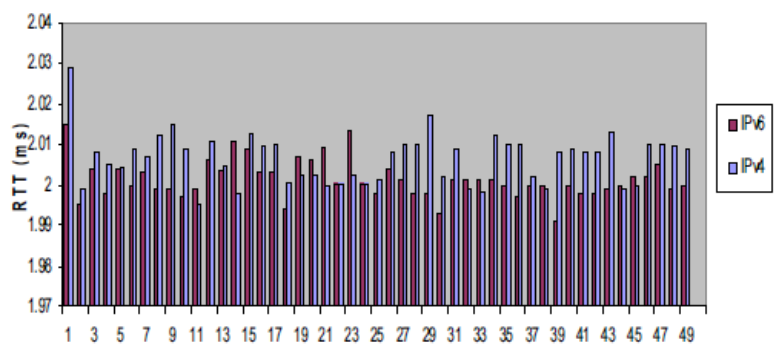

Figure 4: IPv6 and IPv4 network round-trip delay comparison under dual stack

RTT Compare

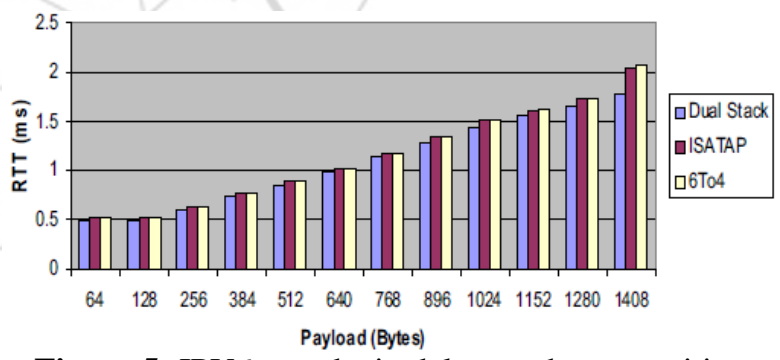

Figure 5: IPV6 round-trip delay on three transition mechanisms

It can be seen from Figure 4, the round-trip delay of IPv6 is shorter than IPv4 with the same network load and packet size for dual stack mechanism, and Figure 5 shows roundtrip delay of 6to 4 tunnel mechanism is similar to ISATAP tunnel mechanism under the same test conditions, but roundtrip delay of IPv6 network for dual stack mechanism is the shortest comparing with 6to4 and ISATAP tunnel mechanism. It should be noted that above test results are obtained in a small Ethernet LAN environment which have some limitations and specificities. The further research should be carried out in the WAN. 


\section{Conclusion}

Based on analysis transition mechanism of IPv6, the dual stack protocol, 6 to 4 tunneling mechanism, and ISATAP tunnel network performance are studied and tested based on program implemented by our group. In general, results indicate that dual stack protocol IPv6 network has better performance than dual stack protocol IPv4, and 6 to 4 mechanism and ISATAP mechanism.

\section{References}

[1] Shaofeng. Scheme for Ipv4/Ipv6 Dual Stack Network. Microcomputer Information.2010-33.

[2] Yang Yi,Dong Yongqiang. Performance Decision on Cost and Delay in IPv6 Networks. Computer Applications and Software. 2010-12.

[3] YANG Zhi-yi, LI Xiao-yan. Study and implementation of IPv4/IPv6 transition technology based on multi-core. Journal of Computer Applications.2009-03.

[4] YANG Zhi-yi, LI Xiao-yan. Study and implementation of IPv4/IPv6 transition technology based on multi-core. Journal of Computer Applications.2009-03.

[5] CHEN jing. Research on Strategy Transformed from IPv4 into IPv6 of Campus Network. Dalian Jiaotong University [D].2010.

[6] RFC2330, Framework for IP Performance Metrics[S].

[7] SU Yun-cheng, SONG Ru-min. Realizes IPv4 to the IPv6 Transition Double Agreement Stack Technology and the Tunnel Technology.Computer Knowledge and Technology.2009-20.

[8]ZHANG Qing, LU Zheng-shan, SHEN Guo-liang. Research and Implementation on Transition Mechanism from IPv4 to IPv6 Based on Campus Network. Journal of Soochow University (Engineering Science Edition).2006-06.

\section{Author Profile}

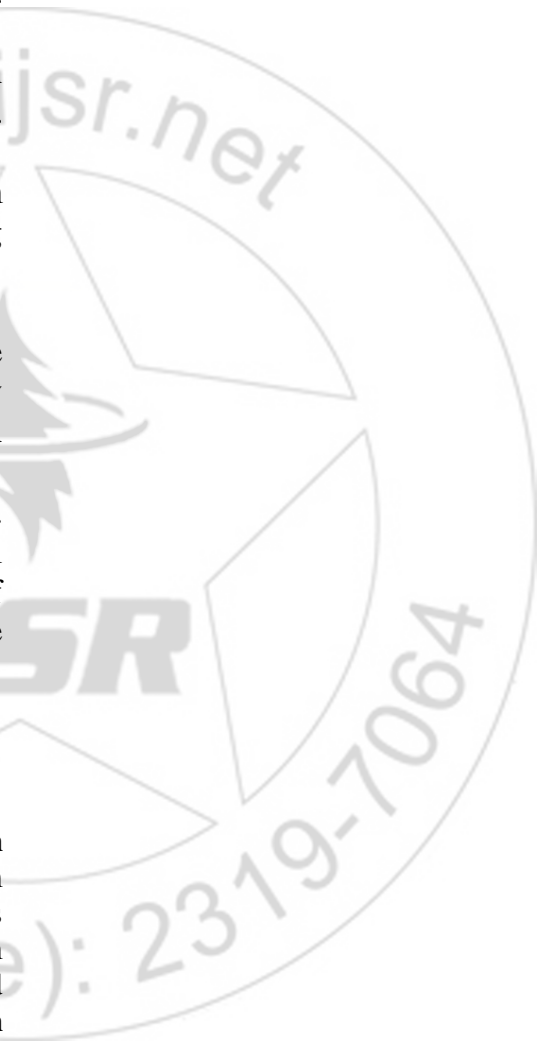

Mr. A. Rajkumar received the B.E degree in electronics and communication engineering from KVCET Engineering College, Chengalpet. $\mathrm{He}$ is currently doing M. E in Communication Systems from Anna University, Thanjavur, India. He has presented papers in international conferences and published papers in international journals.

Mr. Dr. G. Kannan M.E, Ph.D. he has done Ph.D in PRIST University. $\mathrm{He}$ is currently working as a Professor in P.R.Engineering College, Thanjavur, Tamilnadu. Her research area is Visual Communication. 